\title{
Differential expression of osteocalcin during the metastatic progression of prostate cancer
}

\author{
THOMAS A. GARDNER ${ }^{1}$, SANG-JIN LEE ${ }^{2}$, SANG-DON LEE $^{3}$, XIONG LI $^{1}$, TOSHIHIRO SHIRAKAWA ${ }^{4}$, \\ DONG DEUK KWON ${ }^{5,7}$, RA YOUNG PARK ${ }^{6,7}$, KYU YOUN AHN ${ }^{6,7}$ and CHAEYONG JUNG ${ }^{6,7}$ \\ ${ }^{1}$ Department of Urology, Indiana University School of Medicine, Indianapolis, IN 46202, USA; \\ ${ }^{2}$ National Cancer Center, Gyeonggi-do; ${ }^{3}$ Pusan National University Medical School, Pusan, Korea; \\ ${ }^{4}$ International Center for Medical Research and Treatment, Kobe University School of Medicine, Kobe, Japan; \\ Departments of ${ }^{5}$ Urology, ${ }^{6}$ Anatomy, Chonnam National University Medical School, and \\ ${ }^{7}$ Research Institute of Clinical Medicine, Chonnam National University, Gwangju, Korea
}

Received September 18, 2008; Accepted November 13, 2008

DOI: $10.3892 /$ or_00000302

\begin{abstract}
Osteocalcin expression is restricted to osteoblasts and serum osteocalcin level is elevated in metastatic bone tumors including prostate tumors, which predominantly metastasizes to the bone and causes typical osteoblastic lesions. Previously, we have reported that osteocalcin RNA is widely expressed but incompletely spliced in the prostate including prostate tumors. Considering that many studies using osteocalcin-driven gene therapy have been conducted to treat hormone refractory metastatic tumors, detailed mechanisms controlling osteocalcin expression needs to be clarified. We aim to learn how osteocalcin expression is regulated during the metastatic process of prostate cancer. We applied assays of immunohistochemistry and RNA in situ hybridization in prostate tumors acquired from prostate (15) and metastatic sites, 13 from lymph node and 14 from bone. RT-PCR analysis in various cultured prostate cells was also performed. As predicted, osteocalcin RNA was highly expressed in most prostate epithelial cells of tumors, regardless of metastatic status of the tumor. However, osteocalcin protein was undetectable in tumors acquired from the primary site or lymph nodes whereas protein was highly expressed in the majority of bone-metastasized prostate tumors. RT-PCR analysis demonstrated that there was more completely spliced form of osteocalcin RNA present in bone-derived prostate cancer cells. Our data suggest that osteocalcin RNA was expressed but not
\end{abstract}

Correspondence to: Dr Chaeyong Jung, Department of Anatomy, Chonnam National University Medical School, Gwangju, Korea E-mail: chjung@jnu.ac.kr

Abbreviations: OC, osteocalcin; PCa, prostate cancer; IHC, immunohistochemistry; ISH, in situ hybridization

Key words: osteocalcin, prostate cancer, metastasis completely spliced in non-bone environment, ultimately resulting in improper production of osteocalcin protein. This study explains why serum osteocalcin level is increased in patients with bone-metastasized prostate cancers. Yet, it remains to be clarified what regulates bone-specific osteocalcin RNA splicing in prostate tumors.

\section{Introduction}

Human OC is composed of four exons and three introns and codes for 100 amino acid OC precursor, which further cleaves into a mature 51 amino acid peptide and constitutes $1-2 \%$ of the total protein in bone (1). OC functions at later stages of bone formation in the regulation of mineral deposition and turnover of bone. A number of studies showed that OC transcription is enhanced in mature osteoblasts while suppressed in early progenitors (2). Other than bone-related tissues, OC is synthesized by vascular smooth muscle cells and its mRNA is also expressed in megakaryocytes and peripheral blood platelets in rat, which possibly contribute to the OC levels in blood and the regulation of bone turnover (3).

Prostate cancer prevalently metastasizes to the bone and creates a common feature of osteosclerotic lesion with increased osteoblastic activity (4). It has been suggested that bone-metastasized PCa cells are osteomimetic and express genes and proteins which they normally do not express as bone-derived cells do. Since early 1990, several groups have observed that osteocalcin levels were significantly higher in patients with bone metastases whereas high levels were not observed in patients without bone metastases (5-8). High sensitivity and specificity levels of serum osteocalcin appear to be strongly correlated to metastatic bone involvement and disease relapse after hormone treatment. At the same time, Levedakou et al (9) have observed that OC was also highly expressed in primary prostatic carcinomas as compared to prostate cell lines derived from metastatic tumors, and to lymph node metastasis, suggesting that the loss of MGP expression may be associated with tumor progression and metastasis. Nevertheless, it is generally believed that OC 
expression is increased by cis-acting transcription factors at OC promoter in androgen-independent prostate cancers, which are preferably metastasized to bone $(10,11)$. On the other hand, we have previously observed that expression of $\mathrm{OC}$ in non-bone tissues, including normal prostate and prostatic cancer cells, mostly resulted from incomplete splicing event which may prevent proper formation of functional OC proteins (12).

In this study we hypothesized that $\mathrm{OC}$ expression may be altered during the course of prostate cancer progression, in terms of metastasis. To see the differential expression and tissue distribution of OC RNA and protein, assays of in situ hybridization (ISH) and immunohistochemistry (IHC) were performed in localized and metastatic human prostate tumors.

\section{Materials and methods}

Materials. Human prostate tumors were obtained from Department of Pathology at Indiana University. Tumors consisted of 15 local tumors, 13 lymph node-metastasized tumors, and 14 bone-metastasized tumors. Localized tumors were acquired from patients who underwent radical prostatectomy without history of metastasis. Lymph node-metastsized tumors were punch biopsy specimens. Bone-metastasized tumors were acquired though either biopsy or autopsy. All tumors were formalin-fixed and paraffin-embedded.

In situ hybridization (ISH). A set of primers (atttaggtgacacta tacactcctcgecctattggec and taatacgactcactataggggccaactcgtca cagtccgg) was used for developing digoxgenin-labeled riboprobes for OC as described before (12). Labeled riboprobes were synthesized by in vitro transcription of PCR-amplified cDNA using digoxigenin-RNA labeling kit (Roche Biochemicals). RNA ISH was performed on formalin-fixed paraffinembedded human prostate tumor tissues. ISH was performed using $30 \mathrm{ng}$ of probe for each slide. Probes include OC antisense probes, OC sense probes as negative controls, and $B-$ actin probes as positive controls. Briefly, slides were deparaffinized and washed in diethyl pyrocarbonate (DEPC)-treated PBS, followed by DEPC-PBS containing $100 \mathrm{mM}$ glycine. After the sections were treated with proteinase $\mathrm{K}(50 \mu \mathrm{g} / \mathrm{ml})$ (Roche Molecular Biochemicals) at $37^{\circ} \mathrm{C}$ for $30 \mathrm{~min}, 0.25 \%$ acetic anhydride (Sigma) in $100 \mathrm{mM}$ triethanolamine (v/v) was applied for $10 \mathrm{~min}$. The slides were prehybridized with 5X SSC and $40 \%$ deionized formamide at $42^{\circ} \mathrm{C}$ for $30 \mathrm{~min}$ followed by hybridizing with dig-labeled riboprobes in hybridization buffer (40\% deionized formamide, $10 \%$ dextran sulfate, 1X Denhardt's solution, 4X SSC, $10 \mathrm{mM}$ DTT, $1 \mathrm{mg} / \mathrm{ml}$ yeast t-RNA, $1 \mathrm{mg} / \mathrm{ml}$ denatured and sheared salmon sperm DNA) at $42^{\circ} \mathrm{C}$ overnight. Slides were washed twice at $50^{\circ} \mathrm{C}$ in $2 \mathrm{X} \mathrm{SSC} / 0.1 \% \mathrm{SDS}$, then in $1 \mathrm{X} \mathrm{SSC} / 0.1 \%$ SDS, and $0.1 \mathrm{X}$ SSC for $30 \mathrm{~min}$ at room temperature. Signals were then developed using digoxigenin detection method (13).

Immunohistochemistry (IHC). The tissue distribution of OC protein was determined by immunohistochemical staining in fomalin-fixed and paraffin-embedded tissues. After deparaffinzing tissues, slides were microwaved for $10 \mathrm{~min}$ three times in $10 \mathrm{mM}$ citrate buffer ( $\mathrm{pH}$ 6.0) to retrieve antigen. Then, endogenous peroxidase was removed by the treatment of tissues with $0.3 \% \mathrm{H}_{2} \mathrm{O}_{2}$ followed by avidinbiotin blocking. To inhibit non-specific binding of antibodies, slides were treated with $3 \%$ normal goat serum before the incubation of slides with monoclonal anti-OC antibodies (Takara Shuzo Co., OC4-30) overnight. Then, signals were amplified by horseradish peroxidase-DAB detection method.

RT-PCR analysis. Human osteosarcoma MG63 cells and various prostate cancer cell lines were used for the expression of OC RNA. Prostate cancer cells were LNCaP, C4-2, PC3, and MDAPCa2b as well as RWPE normal prostate cells. Cells were routinely cultured in RPMI media supplemented with $5 \% \mathrm{FBS}$ at $37^{\circ} \mathrm{C}$ in an atmosphere of $5 \% \mathrm{CO}_{2}$ before total RNAs were extracted. Total RNAs from each cell line were extracted by using Ultraspec RNA isolation system (Biotecx Laboratories) followed by RNase-free DNase (Promega Corp.) treatment to be free of the possible genomic DNA contamination. Total RNA $(1 \mu \mathrm{g})$ with $100 \mathrm{pM} \mathrm{d}(\mathrm{T}) 20$ was preheated at $72^{\circ} \mathrm{C}$ for $5 \mathrm{~min}$ and reverse transcribed with MMTV RT (Invitrogen) at $42^{\circ} \mathrm{C}$ for $1 \mathrm{~h}$.

A set of OC primers, gaattcatgagagccetcacactcgcc and ctagactagaccgggecgagaagcgecgataggc, was used. Primers for $ß$-actin were used to show the equal loading (5'-gcacca caccttctacaatgagc-3', 5'-tagcacagcctggatagcaacg-3'). PCR was performed in a $50 \mu 1$ solution containing $5 \mu 1$ of $10 \mathrm{X}$ PCR buffer (200 mM Tris-HCl, pH 8.4, $500 \mathrm{mM} \mathrm{KCl),}$ $0.2 \mathrm{mM}$ dNTP mixture, $1 \mu 1$ template DNA, $2 \mathrm{mM} \mathrm{MgCl}_{2}$, $0.2 \mu \mathrm{M}$ of each primer, and 2.5 units of Taq DNA polymerase (Invitrogen). Cycling parameters were: an initial denaturation at $94^{\circ} \mathrm{C}$ for $2 \mathrm{~min}$ followed by 35 cycles with each cycle at $94^{\circ} \mathrm{C}$ for $30 \mathrm{sec}$, at $63^{\circ} \mathrm{C}$ for $30 \mathrm{sec}$, and at $72^{\circ} \mathrm{C}$ for $2 \mathrm{~min}$ and the last extension at $72^{\circ} \mathrm{C}$ for $7 \mathrm{~min}$. PCR products $(5 \mu \mathrm{l})$ were separated by $1 \%$ agarose gel electrophoresis.

\section{Results}

Expression of $O C$ protein is limited to prostate tumor cells metastasized to bone marrow. For the localization of OC, patient samples were acquired through Indiana University. Samples were collected by either biopsy or autopsy. Biopsy samples of bone-metastasized PCa was obtained by bone marrow aspiration followed by cytospin. We have previously shown that OC RNA was abundantly expressed in the normal and cancerous prostate (12). Using RNA ISH, OC was mostly localized to epithelial cells of prostate tumors with moderate expression in stromal cells. However, most of OC RNA expressed in prostate tumors was incompletely spliced forms of OC containing one or more introns, resulting in abnormal expression of proteins. In this study, we used a pool of prostate tumors sorted by metastatic progression to evaluate expression of OC RNA and protein by ISH and IHC, respectively.

To support our hypothesis that OC splicing event is altered during metastatic process of prostate cancer, first, we investigated expression of OC RNA and protein in LNCaP and $\mathrm{C} 42$ prostate cancer cells using ISH and IHC (Fig. 1). LNCaP and C42 cells were previously demonstrated to mostly express incompletely spliced form of OC RNA (12). As we predicted, expression of OC RNA was abundant 

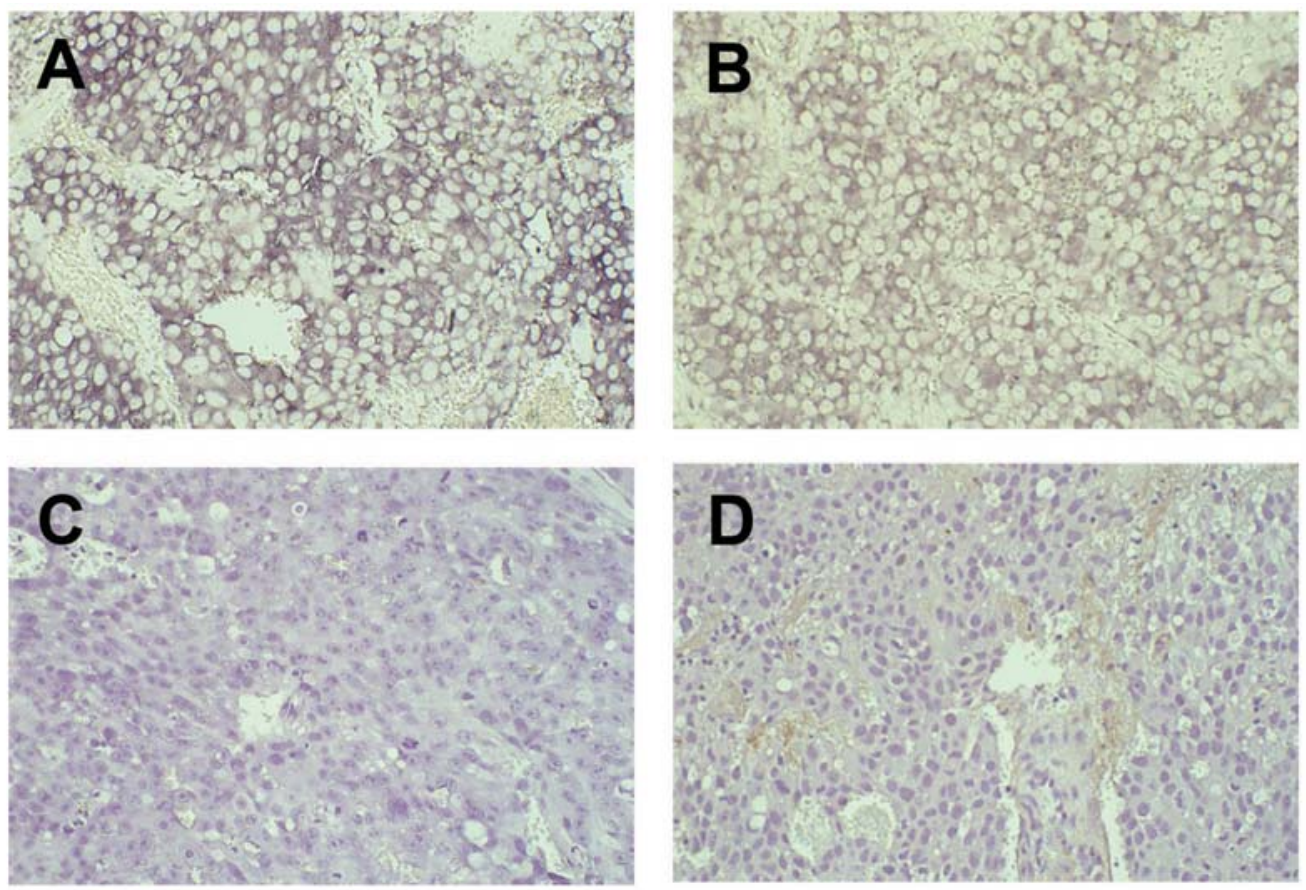

Figure 1. Expression of OC RNA and protein in xenograft tumors. LNCaP and C42 cells were injected into subdermis of nude mice to generate xenograft tumors. Tumors are retrieved from the animals and processed to generate histological samples. Slides then underwent ISH and immunohistochemical procedures. $\mathrm{C}$ and $\mathrm{D}$ were counterstained with hematoxyline.

in both cell types (Fig. 1A and B) while proteins are scant (Fig. 1C and D). This result demonstrates that our approach to detect both RNA and protein molecules can be adequately applied to test our hypothesis in tumors. Further studies have been done on prostate tumors of various metastatic grades (Fig. 2). By ISH, OC riboprobes were hybridized to its counterparts both in epithelial cells and in stromal cells of all prostate tumors, regardless of tumor origin (Fig. 2D-F). In localized tumors, OC RNA expression was high in most of the tumor epithelial cells whereas the OC level was very low in stromal cells (Fig. 2D). In tumors metastasized to lymph node and bone, OC RNA was detectable in prostate tumor cells in corresponding organs (arrows in Fig. 2D and F, respectively). OC probes were also highly reactive to osteoblasts present along the bony trabeculae (arrowheads in Fig. 2F). Some isolated tumor cells from bone marrow aspirates were also highly OC positive (arrow in Fig. 2Ia). OC RNA was non-reactive to OC sense probes in three different origins of tumors (Fig. $2 \mathrm{G}-\mathrm{Ib})$. Serial sections of prostate tumors were used to immunolocalize OC protein using anti-OC monoclonal antibodies. However, OC proteins were not detectable in tumor cells from either localized tumors (Fig. 2J) or lymph node-metastasized tumors (Fig. 2K). As we reported earlier (12), no or low expression of OC in these subsets of tumors were possibly due to the incomplete splicing event of OC. Surprisingly, OC protein was highly expressed in bonemetastasized prostate tumor cells (Fig. 2L). OC was not detectable in any of the tumors by using normal mouse IgG as a negative control (Fig. 2M-O).

Fig. 3 summarizes the staining score of OC RNA and protein by ISH and IHC, respectively. Scores were given as follows: 1, no staining; 2, weak and focal staining; 3 moderate and dispersed staining; 4, strong and extensive staining. Each score was statistically evaluated as mean \pm standard deviation and shown as a bar graph. Overall, most OC RNA was moderately expressed in prostate tumors regardless of the origin of prostate tumors. However, OC protein was not detectable in localized and lymph node metastasized tumors while bone-metastasized tumors express moderate level of OC protein. These results suggest that transcriptional activity of $\mathrm{OC}$ is high in most of prostate tumors regardless of metastatic status but OC translation is somehow disrupted in majority of tumors except the ones in bone environment.

Bone environment affects the expression of OC protein. To demonstrate that expression of OC protein in bone-metastasized prostate tumors is not due to the sequestration of OC from nearby bone cells, we have selected several prostate cancer cells, including PC 3 and MDA PCa 2 b cells, bone-metastasized prostate cancer cells. LNCaP and C42 prostate cancer cells are derived from lymph nodes. RWPE cells are immortalized prostate cells. Extracted total RNA from cells and tissues were analyzed by RT-PCR using primers with its products encompassing exon 1 to exon 4 of OC gene as described (12). As shown in Fig. 4, RNAs from MG63 osteosarcoma cells exclusively express completely spiced form of OC, while RWPE, LNCaP, and C42 cells express variant size of $\mathrm{OC}$, resulting from incomplete splicing event of OC gene. However, PC3 and MDA PCa 2b cells, bone-metastasized prostate cancer cells, express significantly higher amounts of completely spliced form of OC compared to non-bone-derived prostate cells. This result suggests that bone-metastasized prostate cancer cells acquire new phenomenon to completely splice OC RNA, resulting in proper production of OC protein. The result also leads us to predict that bone tissues may provide the proper 

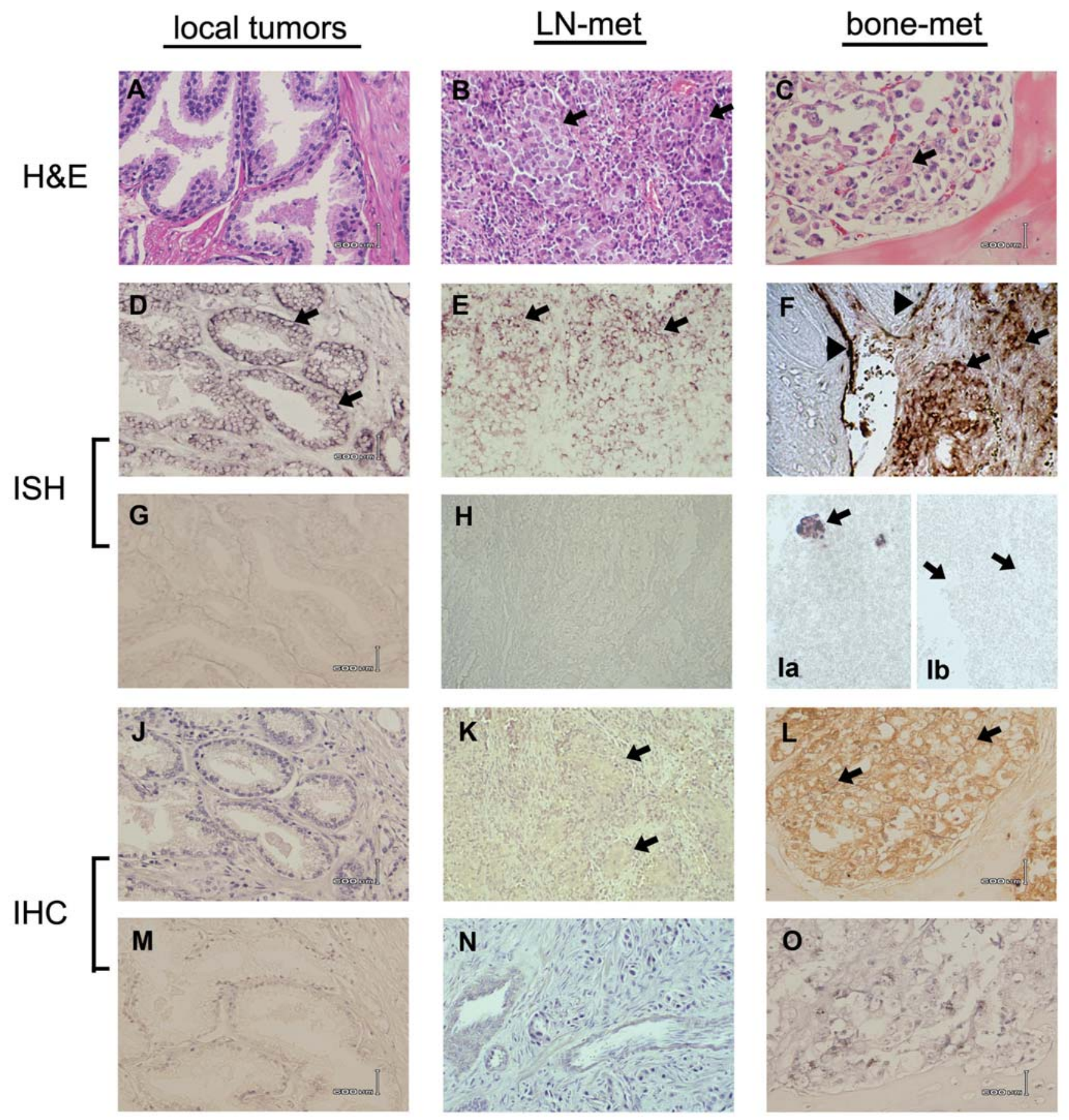

Figure 2. Expression of OC RNA and protein in prostate cancer tumors with various metastatic stage. Tissues with different metastatic origin were processed for detecting OC RNA and protein by ISH and IHC, respectively. (A-C), H\&E stained; (D-I), OC RNA expression by ISH (D-F and Ia with antisense probes, G-H and Ib with sense probes); (J-O), OC protein expression by IHC (J-L with anti-OC antibodies, M-O with normal IgG).

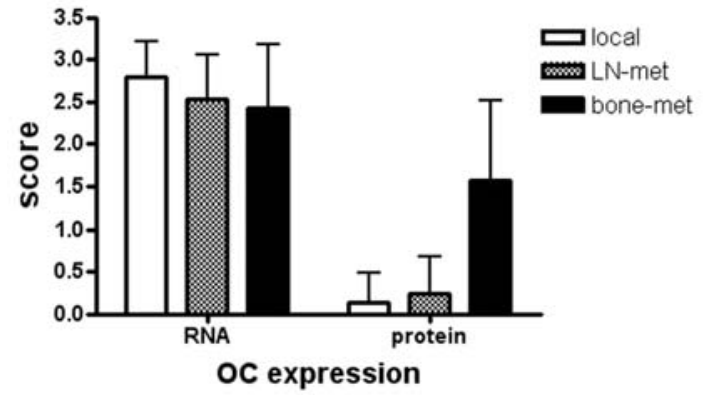

Figure 3. Statistical evaluation of OC RNA and protein expression in tumors. Scored OC expression is shown with mean \pm standard deviation. environment to complete OC splicing by turning on to express a bone tissue-specific splicing factor(s). Expression of OC by prostate cancer cells may stimulate the induction of osteoblastic lesions in the bone, the characteristic phenotype caused by prostate cancer. However, this hypothesis needs to be extensively tested to reveal the interaction between these two cell types.

\section{Discussion}

Osteocalcin is one of the most abundant non-collagenous bone matrix proteins expressed by osteoblasts and is thought 


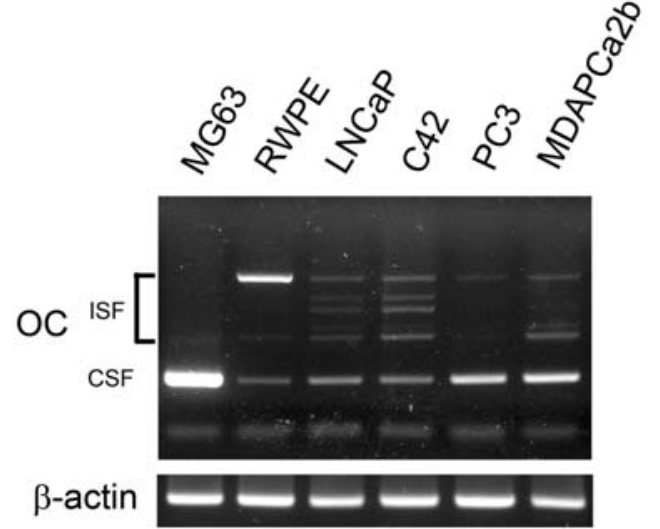

Figure 4. Expression of OC splice variants in cultured cells. Total RNA from cells were analyzed by RT-PCR. PCR products are shown in $1 \%$ agarose gel. ISF, incompletely spliced form; CSF, completely spliced form.

to play a role in osteoblast differentiation and mineralization at late stages of bone formation. Surprisingly, osteocalcin are also expressed by malignant prostate epithelial cells. Prostate tumors preferentially lodge in bone marrow and lead to typical osteoblastic legions. Interaction between prostate cancer cells and bone stromal cells is believed to initiate bone tropism of prostate cancer cells, resulting in the formation of osteoblastic lesions $(5,14-16)$. Growth factors, including fibroblast growth factor 8, vascular endothelial growth factor, receptor activator of nuclear factor kappaB ligand with osteoprotegerin, and activation of extracellular signal-regulated protein kinase (ERK) and notch signaling are involved in this process $(15,17-20)$. Besides, independent activation of ERK and Notch signaling can activate the expression of $\mathrm{OC}$ and generates osteoblastic lesion by prostate cancer cells in bone environment (20). Beta2-microglobulin enhances OC and bone sialoprotein gene expression in human prostate cancer cells by activating a cyclic AMPdependent protein kinase A signaling pathway (21). Galectin-1 is also involved in the osteoblastic response, caused by prostate cancer cells metastasizing into bone, by affecting the matrix mineralization (14). At the same time, prostate-specific antigen produced by metastatic prostate cancer cells may participate in bone remodeling in favor of the development of osteoblastic metastases in the heterogeneous mixture of osteolytic and osteoblastic lesions (22).

Transcription of the murine OC gene is controlled by a complicated mechanism mediated by Runx2 $(23,24)$, Msx2/Dlx 5 homeodomain proteins (25), steroid hormone receptors including glucocorticoid receptor, estrogen receptor, vitamin D receptor, androgen (26-28), TLE co-repressor (29), HDAC3 histone deacetylase, CBP/p300 histone acetyltransferase (30), and SWI/SNF chromatin remodeling factors (31). Generally, OC transcriptional activity using mouse OC promoter is highly active in most prostate cancer cells, regardless of the status of androgen receptor and prostatespecific antigen. In fact, viral gene therapy using OC promoter to control the expression of toxic (therapeutic) gene in a tissue-specific manner is potentially effective to cure metastatic prostate cancers (32-36). We have previously demonstrated that expression of $\mathrm{OC}$ was also regulated by tissuespecific RNA splicing event. We identified incompletely spliced variants of human OC mRNA, existing dominantly in non-osseous organs. Most non-osseous tissues expressed transcripts with higher molecular weight, prominent in ovary, kidney, pancreas, spleen, thymus, prostate, and testis, than the expected size of OC mRNA as seen in bone marrow. Further analyses identified up to six OC transcripts in most tissues tested except bone marrow and most RNA variants contained one or more introns. MG63, an osteoblastic osteosarcoma cell, expressed only the completely-spliced form of OC, whereas incompletely spliced RNA was dominant in most prostate tumor cells (12). In this study, we investigated the presence of OC splicing variants during the course of metastatic process of prostate cancer. As expected, most localized tumors mainly express incompletely spliced forms of OC whereas bone-metastasized tumors acquire completely spliced form of OC and thereby mature form of OC protein. In contrast, prostate tumors metastasized to lymph nodes expressed high level of incomplete form of OC, but did not express OC proteins. Further RT-PCR analysis showed that all prostate cancer cells acquired from bone express significantly higher level of completely spliced form of OC compared to normal prostate cell and tumor cells from lymph nodes. This alternative RNA splicing event of OC may contribute to the acquisition of new osteoblastic activity of prostate cancer cells in bone environment. On the other hand, OC splice variants may provide tropism of prostate cancer cells to the bone, namely metastatic potential to the bone.

In conclusion, the expression of $\mathrm{OC}$ is known to be involved in the progression of prostate cancer metastasis and, in fact, its high serum level is associated with metastatic tumors (5-8). In this study, we demonstrated that prostate tumors express OC RNA but not much OC protein due to incomplete splicing process of OC pre-RNA. A similar result was observed in lymph node-metastasized tumors. Bone-metastasized prostate tumors, however, produce more properly-spliced OC RNA transcripts, which code for functional OC protein. With increasing usage of OC promoter based therapeutics to treat patients with bone-metastasized prostate cancer, the splicing phenomenon of OC and its biological role(s) in bonemetastasized prostate cancer cells need further investigation.

\section{Acknowledgements}

This work was supported by the Korea Science \& Engineering Foundation through the Medical Research Center for Gene Regulation (R13-2002-013-04002-0) at Chonnam National University, and partly by a research grant (CRI07009-1) from the Chonnam National University Hospital Research Institute of Clinical Medicine.

\section{References}

1. Celeste AJ, Rosen V, Buecker JL, Kriz R, Wang EA and Wozney JM: Isolation of the human gene for bone gla protein utilizing mouse and rat cDNA clones. EMBO J 5: 1885-1890, 1986.

2. Hauschka PV, Lian JB, Cole DE and Gundberg CM: Osteocalcin and matrix Gla protein: vitamin K-dependent proteins in bone. Physiol Rev 69: 990-1047, 1989.

3. Thiede MA, Smock SL, Petersen DN, Grasser WA, Thompson DD and Nishimoto SK: Presence of messenger ribonucleic acid encoding osteocalcin, a marker of bone turnover, in bone marrow megakaryocytes and peripheral blood platelets. Endocrinology 135: 929-937, 1994. 
4. Goltzman D: Mechanisms of the development of osteoblastic metastases. Cancer 80: 1581-1587, 1997.

5. Arai Y, Takeuchi H, Oishi K and Yoshida O: Osteocalcin: is it a useful marker of bone metastasis and response to treatment in advanced prostate cancer? Prostate 20: 169-177, 1992.

6. Curatolo C, Ludovico GM, Correale M, et al: Advanced prostate cancer follow-up with prostate-specific antigen, prostatic acid phosphatase, osteocalcin and bone isoenzyme of alkaline phosphatase. Eur Urol 21 (Suppl. 1): 105-107, 1992.

7. Marcellini M, De Carli P, Abbolito MR, Mainiero G and Cantiani R: Serum osteocalcin in monitoring bone metastases in advanced prostatic cancer. Eur Urol 21 (Suppl. 1): 102-104, 1992

8. Shih WJ, Wierzbinski B, Collins J, Magoun S, Chen IW and Ryo UY: Serum osteocalcin measurements in prostate carcinoma patients with skeletal deposits shown by bone scintigram: comparison with serum PSA/PAP measurements. J Nucl Med 31: 1486-1489, 1990.

9. Levedakou EN, Strohmeyer TG, Effert PJ and Liu ET: Expression of the matrix Gla protein in urogenital malignancies. Int J Cancer 52: 534-537, 1992

10. Yeung F and Chung LW: Molecular basis of co-targeting prostate tumor and stroma. J Cell Biochem Suppl 38: 65-72, 2002.

11. Yeung F, Law WK, Yeh CH, et al: Regulation of human osteocalcin promoter in hormone-independent human prostate cancer cells. J Biol Chem 277: 2468-2476, 2002.

12. Jung C, Ou YC, Yeung F, Frierson HF Jr and Kao C: Osteocalcin is incompletely spliced in non-osseous tissues. Gene 271: 143-150, 2001

13. Herrington CS, Troncone G, Evans MF and McGee JO: Screening for high- and low-risk human papillomavirus types in single routine cervical smears by non-isotopic in situ hybridization. Cytopathology 3: 71-78, 1992.

14. Andersen H, Jensen ON, Moiseeva EP and Eriksen EF: A proteome study of secreted prostatic factors affecting osteoblastic activity: galectin-1 is involved in differentiation of human bone marrow stromal cells. J Bone Miner Res 18: 195-203, 2003.

15. Dai J, Kitagawa Y, Zhang J, et al: Vascular endothelial growth factor contributes to the prostate cancer-induced osteoblast differentiation mediated by bone morphogenetic protein Cancer Res 64: 994-999, 2004.

16. Koeneman KS, Yeung F and Chung LW: Osteomimetic properties of prostate cancer cells: a hypothesis supporting the predilection of prostate cancer metastasis and growth in the bone environment. Prostate 39: 246-261, 1999.

17. Kitagawa Y, Dai J, Zhang J, et al: Vascular endothelial growth factor contributes to prostate cancer-mediated osteoblastic activity. Cancer Res 65: 10921-10929, 2005.

18. Valta MP, Hentunen T, Qu Q, et al: Regulation of osteoblast differentiation: a novel function for FGF-8. Endocrinology, 2006.

19. Valta MP, Tuomela J, Bjartell A, Valve E, Vaananen HK and Harkonen P: FGF-8 is involved in bone metastasis of prostate cancer. Int J Cancer 123: 22-31, 2008

20. Zayzafoon M, Abdulkadir SA and McDonald JM: Notch signaling and ERK activation are important for the osteomimetic properties of prostate cancer bone metastatic cell lines. J Biol Chem 279: 3662-3670, 2004.

21. Huang WC, Wu D, Xie Z, et al: Beta2-microglobulin is a signaling and growth-promoting factor for human prostate cancer bone metastasis. Cancer Res 66: 9108-9116, 2006.

22. Nadiminty N, Lou W, Lee SO, et al: Prostate-specific antigen modulates genes involved in bone remodeling and induces osteoblast differentiation of human osteosarcoma cell line SaOS-2. Clin Cancer Res 12: 1420-1430, 2006.
23. Montecino M, Lian J, Stein G and Stein J: Changes in chromatin structure support constitutive and developmentally regulated transcription of the bone-specific osteocalcin gene in osteoblastic cells. Biochemistry 35: 5093-5102, 1996.

24. Frendo JL, Xiao G, Fuchs S, Franceschi RT, Karsenty G and Ducy P: Functional hierarchy between two OSE2 elements in the control of osteocalcin gene expression in vivo. $\mathrm{J}$ Biol Chem 273: 30509-30516, 1998.

25. Hassan MQ, Javed A, Morasso MI, et al: Dlx3 transcriptional regulation of osteoblast differentiation: temporal recruitment of Msx2, Dlx3, and Dlx5 homeodomain proteins to chromatin of the osteocalcin gene. Mol Cell Biol 24: 9248-9261, 2004.

26. Javed A, Gutierrez S, Montecino M, et al: Multiple Cbfa/AML sites in the rat osteocalcin promoter are required for basal and vitamin D-responsive transcription and contribute to chromatin organization. Mol Cell Biol 19: 7491-7500, 1999.

27. Lian JB, Stein JL, Stein GS, et al: Contributions of nuclear architecture and chromatin to vitamin D-dependent transcriptional control of the rat osteocalcin gene. Steroids 66: 159-170, 2001.

28. Paredes R, Arriagada G, Cruzat F, et al: The Runx 2 transcription factor plays a key role in the 1alpha,25-dihydroxy Vitamin D3-dependent upregulation of the rat osteocalcin (OC) gene expression in osteoblastic cells. J Steroid Biochem Mol Biol 89-90: 269-271, 2004.

29. Javed A, Guo B, Hiebert S, et al: Groucho/TLE/R-esp proteins associate with the nuclear matrix and repress RUNX (CBF (alpha)/AML/PEBP2(alpha)) dependent activation of tissue-specific gene transcription. J Cell Science $113(\mathrm{Pt} \mathrm{12)}$ : 2221-2231, 2000.

30. Sierra J, Villagra A, Paredes R, et al: Regulation of the bonespecific osteocalcin gene by p300 requires Runx $2 / \mathrm{Cbfa} 1$ and the vitamin D3 receptor but not p300 intrinsic histone acetyltransferase activity. Mol Cell Biol 23: 3339-3351, 2003.

31. Villagra A, Cruzat F, Carvallo L, et al: Chromatin remodeling and transcriptional activity of the bone-specific osteocalcin gene require CCAAT/enhancer-binding protein beta-dependent recruitment of SWI/SNF activity. J Biol Chem 281: 22695-22706, 2006.

32. Koeneman KS, Kao C, Ko SC, et al: Osteocalcin-directed gene therapy for prostate-cancer bone metastasis. World J Urol 18: 102-110, 2000.

33. Kubo H, Gardner TA, Wada Y, et al: Phase I dose escalation clinical trial of adenovirus vector carrying osteocalcin promoterdriven herpes simplex virus thymidine kinase in localized and metastatic hormone-refractory prostate cancer. Hum Gene Ther 14: 227-241, 2003.

34. Matsubara S, Wada Y, Gardner TA, et al: A conditional replication-competent adenoviral vector, Ad-OC-E1a, to cotarget prostate cancer and bone stroma in an experimental model of androgen-independent prostate cancer bone metastasis. Cancer Res 61: 6012-6019, 2001.

35. McCarthy HO, Coulter JA, Worthington J, Robson T and Hirst DG: Human osteocalcin: a strong promoter for nitric oxide synthase gene therapy, with specificity for hormone refractory prostate cancer. J Gene Med 9: 511-520, 2007.

36. Shirakawa T, Terao S, Hinata N, et al: Long-term outcome of phase I/II clinical trial of Ad-OC-TK/VAL gene therapy for hormone-refractory metastatic prostate cancer. Hum Gene Ther 18: 1225-1232, 2007. 